\title{
Rocking Subdiffusive Ratchets: Origin, Optimization and Efficiency
}

\author{
I. Goychuk ${ }^{1 *}$, V. O. Kharchenko ${ }^{2}$ \\ ${ }^{1}$ Institute of Physics, University of Augsburg, Universitätstr. 1, D-86135 Augsburg, Germany \& \\ Institute for Physics and Astronomy, University of Potsdam, Karl-Liebknecht-Str. 24/25, \\ D-14476 Potsdam-Golm, Germany \\ ${ }^{2}$ Institute of Applied Physics, NAS of Ukraine, 58 Petropavlovskaya str., 40030 Sumy, Ukraine
}

\begin{abstract}
We study origin, parameter optimization, and thermodynamic efficiency of isothermal rocking ratchets based on fractional subdiffusion within a generalized non-Markovian Langevin equation approach. A corresponding multi-dimensional Markovian embedding dynamics is realized using a set of auxiliary Brownian particles elastically coupled to the central Brownian particle (see video on the journal web site). We show that anomalous subdiffusive transport emerges due to an interplay of nonlinear response and viscoelastic effects for fractional Brownian motion in periodic potentials with broken space-inversion symmetry and driven by a time-periodic field. The anomalous transport becomes optimal for a subthreshold driving when the driving period matches a characteristic time scale of interwell transitions. It can also be optimized by varying temperature, amplitude of periodic potential and driving strength. The useful work done against a load shows a parabolic dependence on the load strength. It grows sublinearly with time and the corresponding thermodynamic efficiency decays algebraically in time because the energy supplied by the driving field scales with time linearly. However, it compares well with the efficiency of normal diffusion rocking ratchets on an appreciably long time scale.
\end{abstract}

Keywords and phrases: anomalous Brownian motion, generalized Langevin equation, memory effects, viscoelasticity, ratchet transport, stochastic thermodynamics

Mathematics Subject Classification: 82C31, 82C70, 82C80, 60H10

\section{Introduction}

Theories of anomalous Brownian motion stem from pioneering contributions by Kolmogorov [34], Mandelbrot and van Ness [43], Montroll and Weiss [48], Shlesinger [54], Scher and Montroll [51], and others. Mandelbrot and van Ness coined first the notion of fractional Brownian motion (fBm) featured by long range interdependence of the particle position increments, although $\mathrm{fBm}$ was implicitly introduced earlier by Kolmogorov as a class of curves in an abstract Hilbert space. They considered a Riemann-Liouville

${ }^{*}$ Corresponding author. E-mail: goychuk@physik.uni-augsburg.de 
fractional integral of the white Gaussian noise $\xi(t)=d B(t) / d t$, or the Holmgren-Riemann-Liouville fractional integral of normal Brownian motion $B(t)$,

$$
B_{H}\left(t, t_{0}\right)=\frac{\tau_{0}^{-H+1 / 2}}{\Gamma(H+1 / 2)} \int_{t_{0}}^{t}\left(t-t^{\prime}\right)^{H-1 / 2} \xi\left(t^{\prime}\right) d t^{\prime},
$$

with $t_{0} \rightarrow-\infty$ to ensure the stationarity of increments. The parameter $H$ is the Hurst exponent $0<H<1$ and an arbitrary time scaling factor $\tau_{0}$ guarantees that the physical dimension of $B_{H}$, e.g. that of coordinate $x=B_{H}$, is the same for any $H$. Such an integral diverges, but the difference $B_{H}(t):=B_{H}(t,-\infty)-B_{H}(0,-\infty)$ remains finite. It serves as a definition for $\mathrm{fBm}$. The variance of $\mathrm{fBm}$ scales in time as $\operatorname{Var}\left(B_{H}(t)\right) \propto t^{\alpha}$. Here, $\alpha=2 H$ is the power exponent of anomalous diffusion. The self-similar scaling of normal Brownian motion, i.e. the fact that the processes $h^{-1 / 2} B(h t)$ and $B(t)$ are identical in distribution implies akin equivalence between the processes $h^{-H} B(h t)$ and $B_{H}(t)$, in accordance with the above definition. The case of $H=1 / 2(\alpha=1)$ corresponds to normal diffusion with independent position increments. Slowly decaying negative correlations for $0<H<1 / 2$ lead to subdiffusion, $0<\alpha<1$, while the positive ones for $1 / 2<H<1$ yield superdiffusion, $1<\alpha<2$.

Fractional Brownian motion can emerge $[19,21,24]$ as a solution of the generalized Langevin equation or GLE $[35,36,60,61]$

$$
m \ddot{x}+\int_{-\infty}^{t} \eta\left(t-t^{\prime}\right) \dot{x}\left(t^{\prime}\right) d t^{\prime}=f(x, t)+\xi(t)
$$

where the memory kernel $\eta(t)$ and colored zero-mean Gaussian noise $\xi(t)$ obey the fluctuation-dissipation relation,

$$
\left\langle\xi(t) \xi\left(t^{\prime}\right)\right\rangle=k_{B} T \eta\left(\left|t-t^{\prime}\right|\right)
$$

at the temperature $T$ which characterizes the thermal environment and the intensity of the thermal noise it produces. Brackets denote averaging over the noise realizations, and $k_{B}$ is the Boltzmann constant. The following conditions are required for this: (1) The corresponding noise is fractional Gaussian noise (fGn) defined as the derivative of $\mathrm{fBm}, \xi(t)=\dot{B}_{1-\alpha / 2}(t) .{ }^{1}(2)$ The memory kernel is accordingly

$$
\eta(t)=\frac{\eta_{\alpha}}{\Gamma(1-\alpha)} t^{-\alpha}
$$

for $0<\alpha<1$, where $\Gamma(z)$ is the gamma-function. For $1<\alpha<2$ and $t>0$, one defines the memory kernel by the same expression, which takes on negative values. However, there is a positive singularity at $t \rightarrow 0$, so that the integral of the memory friction is always positive for such a superdiffusive GLE and it tends to zero with the upper integration limit $t \rightarrow \infty$. (3) The inertial effects are negligible, $m \rightarrow 0$ ( $m$ is the mass of Brownian particle), and (4) an external regular force is absent, $f(x, t) \rightarrow 0$. This particular model is also known under the label of fractional Langevin equation (FLE) [19, 39, 41] upon the formal use of the operators of fractional derivative to abbreviate the frictional memory term. The physical origin of subdiffusion for $0<\alpha<1$ is viscoelasticity [21,24]. The memoryless kernel $\eta(t)=2 \eta_{1} \delta(t)$, where $\eta_{1}$ is the viscous friction coefficient, yields normal Brownian motion. It corresponds to a viscous Newtonian fluid, which can be characterized by a macroscopic viscosity coefficient $\zeta, \eta_{1} \propto \zeta$. Another extreme of non-decaying memory kernel, $\eta(t) \approx$ const, corresponds to a quasi-elastic trapping force. Maxwell was first [46] to discuss within a purely macroscopic setting, which neglects fluctuations, the emergence of the phenomenon of viscosity of fluids from the elasticity of solids by letting the elastic deformation forces relax exponentially in time. This corresponds to a simple viscoelastic fluid, which can be characterized by a viscosity kernel exponentially decaying in time. The corresponding generalized Brownian motion is characterized by the memory kernel $\eta(t)=\kappa \exp (-\nu t)$, where $\kappa$ is an elastic constant

\footnotetext{
${ }^{1}$ Notable is that the superdiffusive persistent noise source in GLE (1.2) yields anti-persistent subdiffusion as the GLE solution and vice versa. This is due to the influence of memory kernel, integral of which diverges for subdiffusion and is zero for superdiffusion, in the limit $t \rightarrow \infty$.
} 
and $\nu$ is a corresponding relaxation rate. Gemant found [14] that power law dependences like one in (1.3) describe well a number of complex viscoelastic materials. Benchmark of a complex fluid is, however, that its macroscopic viscosity is finite. This implies that a total integral of the memory kernel $\tilde{\eta}(0)$, where $\tilde{\eta}(s)=\int_{0}^{t} \exp (-s t) \eta(t) d t$ is the Laplace-transformed memory kernel, is finite as well. Therefore, a long range memory cutoff $t_{\mathrm{h}}$ is necessarily present for any liquid-like matter. The existence of an upper bound $\omega_{\mathrm{h}}$ for the frequency of vibrational modes of any medium implies also the existence of a short memory cutoff $t_{1} \propto 1 / \omega_{\mathrm{h}}$. For this reason, a power law scaling leading to subdiffusion always extends in nature only over some finite number, $N_{\max }=\log _{10}\left(t_{\mathrm{h}} / t_{1}\right)$, of time, or frequency decades, and anomalous Brownian motion is mostly a transient phenomenon. However, it can be a dominant mechanism of transport and diffusion on mesoscale [25] which makes the mathematical models of fBm and FLE very important. Any fractal scaling in nature is restricted by a certain maximal spatial and/or time range, mostly over several decades only, seldomly over six or more spatial, or time decades. This fact does not undermine, however, grandeur and usefulness of the fractal type modeling approaches [44].

Montroll and Weiss [48], Shlesinger [54], and Scher [51] proposed a different description of anomalous Brownian motion based on continuous time random walks (CTRWs) with a heavy-tail distribution of the residence times spent on the sites of localization before making a spatial jump to a different localized state. Such a CTRW is semi-Markovian, i.e. the residence times on different sites are independently distributed and the spatial steps are not correlated, but the first moment of the residence time distribution (RTD) can diverge, as e.g. for $\psi(\tau) \propto \tau^{-1-\alpha}, 0<\alpha<1$. This divergence gives rise to subdiffusion, if the spatial distribution of the particle's jumps has first two moments finite. Such a subdiffusion mechanism can be related to a spatial and/or energy disorder. Namely, RTDs are exponential and can be characterized by a spatially dependent transition rate. However, the escape rates out of different localized traps are vastly different due to disorder, so that the averaging of the RTD over disorder yields an effective RTD with divergent mean time. In the effective medium or mean-field approximation, the later approach is equivalent to a semi-Markovian CTRW [6,31]. Then, a spatial disorder is equivalent to a stochastic clock with vastly varying but independent time intervals elapsed between jump events (as usually, mean field approaches neglect correlations). In the continuous space limit, this another anomalous Brownian motion can be described by the fractional diffusion equation and its extension to the diffusion in external force-fields, the fractional Fokker-Planck equation [3,47]. It is related to normal Brownian motion in accordance with the concept of subordination $[3,55]$. One can obtain it from the normal one by replacing the physical time $t$ with a stochastic intrinsic time $\tau(t), B(t) \rightarrow B(\tau(t))$. $B(\tau(t))$ is thus a doubly stochastic process. Intrinsic stochastic time, or the particle's "age" is measured by a number of random clock periods accomplished within the time interval $[0, t)$. Notice that the occurrence of very long time periods implies smaller $\tau$. For this reason the average of $\tau$ exists and it scales with physical time as $\langle\tau\rangle \propto t^{\alpha}$. This random time is described by a probability density $p_{\alpha}(\tau \mid t)=\frac{t}{\alpha \tau^{1+1 / \alpha}} \mathcal{L}_{\alpha}\left(t / \tau^{1 / \alpha}\right)$, where $\mathcal{L}_{\alpha}(x)$ is the probability density of one-sided Levy stable distribution $[3,23,27,57]$. For normal diffusion, $p_{1}(\tau \mid t)=\delta(t-\tau)$, i.e. $\tau$ is not a random variable. It has the fixed value $t$. In an infinite medium, the averaging over one particle trajectory yields a normal diffusion scaling of the position variance with time. It can be characterized by a normal diffusion coefficient which, however, depends on the time interval of observation and algebraically decays upon the observation time increase [27,38]. Different trajectories are characterized by different normal diffusion coefficients, but the ensemble averaging yields subdiffusion. This indicates clearly a breaking of ergodicity and is very different from the case of fBM subdiffusion, where a single-particle trajectory averaging yields subdiffusive behavior for each particle separately, and which is clearly ergodic $[10,21]$. Averaging of such a doubly stochastic process over the realizations of $\tau$ yields a random process

$$
\left\langle B_{\alpha, \mathrm{CTRW}}(t)\right\rangle_{\tau}=\int_{0}^{\infty} p_{\alpha}(\tau \mid t) B(\tau) d \tau .
$$

It is very different from $\mathrm{fBm}$, even though the scaling of its variance with time is virtually the same.

In the focus of this work is the noise-assisted anomalous subdiffusive ratchet transport [22] in a spatially periodic force-field with broken space inversion symmetry and driven in addition by a periodic external 
force $[4,40,45,50]$. At thermal equilibrium, the net transport is absent because the symmetry of thermal detailed balance forbids such a transport if only the transport is not induced by a traveling potential trapping the particle and carrying it on. The latter transport has almost mechanical character and is not induced but rather hindered by thermal fluctuations. It is not considered here. Furthermore, the noise-assisted ratchet transport is absent in the linear response regime to periodic driving due to the Onsager symmetry of kinetic coefficients for a nearly equilibrium transport. A net rectification current can emerge only in a strongly nonlinear and non-equilibrium regime of driven transport. This general circle of problems is known under the label of Brownian ratchets physics [45,50]. A huge number of papers is devoted to normal diffusion ratchets as reviewed in $[45,50]$. However, a generalization to the domain of anomalous diffusion is highly nontrivial. Physics of such anomalous Brownian motors is currently still in its infancy. The problem is that within a CTRW subdiffusion scenario based on the RTDs with divergent mean trapping time the asymptotic response to a periodic driving is absent $[2,29,30,56]$, contrary to some published papers which implicitly assumed that the external field oscillates in the intrinsic random time $\tau$ and not the laboratory time $t$. This death of response not only in the linear response regime [56] but also beyond $[29,30]$ makes the fluctuating tilt or rocking ratchets based on the CTRW subdiffusion barely possible. However, the viscoelastic subdiffusion of the fBm type also displays a remarkable feature which makes the emergence of rectification effect highly nontrivial. Namely, neither diffusion nor transport depend asymptotically on the presence of a periodic potential [21,23,24]. This makes ratchet effect for an adiabatically slow driving with vanishingly small frequency clearly impossible. The transient to this asymptotic transport regime is, however, very slow. It strongly depends, in particular, on the amplitude of the periodic potential and temperature $[21,23,24]$ which makes anomalous ratchet effect possible in a nonadiabatic driving regime [22]. Moreover, the anomalous transport optimizes when the periodic tilts synchronize with an intrinsic time scale of activated transitions to nearest potential wells [26]. An optimal anomalous transport occurs as a manifestation of non-Markovian stochastic resonance [12,15-17]. Moreover, it is a genuine ratchet effect since the transport persists against a sufficiently small loading force and there exists a critical stopping force which depends on the model parameters [26].

In this paper, we continue to investigate the optimization of anomalous transport depending on the model parameters such as temperature, potential height, frequency and strength of the driving field. Moreover, we address for the first time the question of thermodynamic efficiency of anomalous isothermal Brownian motors.

\section{Theory}

A nice feature of $\mathrm{fBm}$ and its generalization, which includes inertial effects and action of external forces, is that it can be derived from a purely dynamical model following to the reductionist type dynamical approach by Bogolyubov [5], Ford, Kac, and Masur [11], Zwanzig [60], and others. Indeed, GLE (1.2) follows from a purely Hamiltonian model of a transport particle moving in a time-dependent potential $V(x, t)$ and coupled elastically with spring constants $k_{i}$ to the harmonic oscillators with masses $m_{i}$, $H=p^{2} /(2 m)+V(x, t)+\sum_{i}\left[p_{i}^{2} /\left(2 m_{i}\right)+k_{i}\left(x-x_{i}\right)^{2} / 2\right]$. This set of oscillators models the influence of environment, or thermal bath, see in $[24,37,59,61]$ for details. The only non-dynamical element in this derivation is that the initial values of environmental degrees of freedom $\left\{p_{i}(0), x_{i}(0)\right\}$ are random and canonically distributed. It is, however, quite in the spirit of modern molecular dynamics. The derivation requires a quasi-continuum of oscillators with dense spectrum to ensure that the noise correlation function asymptotically decays in time to zero. Such a hyper-dimensional embedding dynamics leading to a lowdimensional but non-Markovian GLE dynamics is obviously Markovian. Projection of a multidimensional Markovian dynamics onto a subspace yields typically non-Markovian dynamics of reduced dimensionality. This makes the whole idea of a finite-dimensional stochastic Markovian embedding of the GLE dynamics obvious. Indeed, let us following to [24] consider dynamics of the central particle coupled to a finite set of harmonic Brownian oscillators modeling the environment. The corresponding set of Langevin equations 
reads:

$$
\begin{aligned}
m \ddot{x} & =f(x, t)-\sum_{i=1}^{N} k_{i}\left(x-x_{i}\right), \\
m_{i} \ddot{x}_{i} & =k_{i}\left(x-x_{i}\right)-\eta_{i} \dot{x}_{i}+\sqrt{2 \eta_{i} k_{B} T} \xi_{i}(t),
\end{aligned}
$$

where $\xi_{i}(t)$ are independent unbiased white Gaussian noise sources of unit intensity, $\left\langle\xi_{i}(t) \xi_{j}\left(t^{\prime}\right)\right\rangle=\delta_{i j} \delta(t-$ $t^{\prime}$ ), and $f(x, t)=-\partial V(x, t) / \partial x$. Unlike in the purely dynamical model, we (i) took only a finite set of effective medium's oscillators, and (ii) replaced the rest by the Stokes frictional forces with frictional coefficients $\eta_{i}$, and the corresponding thermal noises acting on these representative Brownian quasiparticles. Furthermore, let us (iii) neglect the inertial effects for the medium's degrees of freedom, $m_{i} \rightarrow 0$, and introduce the (visco)elastic forces $u_{i}=-k_{i}\left(x-x_{i}\right)$ acting on the central particle. Then, the stochastic dynamics in $(2.1)$ reduces to [21]

$$
\begin{aligned}
\dot{x} & =v, \\
m \dot{v} & =f(x, t)+\sum_{i=1}^{N} u_{i}(t), \\
\dot{u}_{i} & =-k_{i} v-\nu_{i} u_{i}+\sqrt{2 \nu_{i} k_{i} k_{B} T} \xi_{i}(t),
\end{aligned}
$$

where $\nu_{i}=k_{i} / \eta_{i}$ are the relaxation rate constants of viscoelastic forces. The last equation in (2.2) recalls an equation for the relaxation of elastic force introduced by Maxwell [46] in his pioneering discussion of macroscopic viscoelasticity. It is augmented by the corresponding Langevin force accounting for thermal fluctuations, in accordance with the fluctuation-dissipation theorem [35]. Therefore, this model can be named generalized Maxwell-Langevin model of viscoelastic stochastic dynamics. It is easy to show that the exclusion of the dynamics of $u_{i}(t)$ variables assuming that the initial $u_{i}(0)$ are Gaussian distributed, unbiased and with the variance $\left\langle u_{i}^{2}(0)\right\rangle=k_{i} k_{B} T$ leads to GLE (1.2), where $\dot{x}(t)=0$ for $t<0$, i.e. the Brownian particle starts to move at $t_{0}=0$, and the memory kernel presents a sum of exponentials:

$$
\eta(t)=\sum_{i=1}^{N} k_{i} \exp \left(-\nu_{i} t\right) .
$$

For such a memory kernel, the Markovian embedding (2.2) of non-Markovian GLE dynamics is exact. However, even a power-law dependence in (1.4) can be approximated by (2.3) between two time cutoffs $t_{l}$ and $t_{h}$ with a controllable accuracy, cf. Fig. 1,a. Indeed, it suffices to take for this $\nu_{i}=\nu_{0} / b^{N-1}$ and $k_{i} \propto \nu_{i}^{\alpha}$ [49]. Here, $\nu_{0} \sim 1 / t_{l}$ is a high-frequency cutoff, and the parameter $b$ controls the accuracy of approximation. With this choice it is easy to show that the corresponding sum $\eta\left(t, \nu_{0}, b, N\right)$ (which depends formally on $\nu_{0}, b$, and $N$ ) has the scaling property, $\eta\left(h t, \nu_{0}, b, N\right)=h^{-\alpha} \eta\left(t, h \nu_{0}, b, N\right)$. The "scale-free" power-law dependence obeys the scaling $\eta(h t)=h^{-\alpha} \eta(t)$, which establishes the meaning of "scale-free". The approximation has indeed this property provided that it does not depend on $\nu_{0}$. Of course, this can only be valid for $t$ far away from the cutoff boundary values. However, the same is valid also for any power-law fractal dependence with cutoffs. The absolute error of approximation (which exhibits logarithmic oscillation, see in Fig. 1,b must also be negligibly small. Indeed, for $\alpha=1 / 2$ and $b=10$, the maximal error of the kernel approximation is about $4 \%$, which already suffices for most stochastic simulations, where $5 \%$ accuracy is a "golden standard". For $b=5$, the maximal error reduces to $0.7 \%$ and for $b=2$ becomes smaller than $0.01 \%$, cf. Fig. 1,b. The nice feature is that the number of exponentials required to approximate the power law over $r$ time decades scales as $N \sim r / \log _{10} b$. The weak dependences on $r$ and $b$ ensure an excellent numerical approach to integrate fractional Langevin dynamics on any time scale of practical interest with a well controlled accuracy. Instead of integrating stochastic integro-differential GLE equation with long-range correlated fractional Gaussian noise, where unfortunately no well-established mathematical results are known on the convergency and accuracy of 
the existing stochastic integration algorithms, we replaced it by the stochastic simulations of an extended multi-dimensional Markovian dynamics with very reliable numerical methods at use. This is probably the best numerical approach at present, which allows to simulate very long trajectories. Practically we used stochastic Heun algorithm for SDEs [13] (second order stochastic Runge-Kutta method). The approach was tested, see in Fig. 2, against the exact solution of the FLE for the potential-free subdiffusion [39],

$$
\left\langle\delta x^{2}(t)\right\rangle=2 v_{T}^{2} t^{2} E_{2-\alpha, 3}\left[-\left(t / \tau_{v}\right)^{2-\alpha}\right] .
$$

Here, $E_{\gamma, \beta}(z)=\sum_{n=0}^{\infty} z^{n} / \Gamma(n \gamma+\beta)$ is the generalized Mittag-Leffler function, $v_{T}=\sqrt{k_{B} T / m}$ is thermal velocity, and $\tau_{v}=\left(\mathrm{m} / \eta_{\alpha}\right)^{1 /(2-\alpha)}$ is a time constant of the anomalous relaxation of equilibrium velocity autocorrelation function, $\left\langle v(t) v\left(t^{\prime}\right)\right\rangle=v_{T}^{2} E_{2-\alpha}\left[-\left(\left|t-t^{\prime}\right| / \tau_{v}\right)^{2-\alpha}\right]$, where $E_{\gamma}(z):=E_{\gamma, 1}(z)$ is the Mittag-Leffler function. In simulations, we scale time in units of $\tau_{v}$, and distance in arbitrary units of $L$ (which becomes the spatial period in the case of periodic potentials). Then, the velocity scales in units of $L / \tau_{v}$, energy in units of $m L^{2} / \tau_{v}^{2}$, force in units of $m L / \tau_{v}^{2}$ and temperature in units of $m L^{2} /\left(\tau_{v}^{2} k_{B}\right)$. We used Markovian embedding with $b=10$ and $N=12, \nu_{0}=100$, time step $\Delta t=0.002$, and $n=10^{4}$ particles in simulations for doing ensemble averaging. Temperature was variable. Simulations were done with double precision on a Fermi NVIDIA graphical processor unit (GPU) which reduced the computational time by a factor of about 100 with respect to standard CPU computing. The agreement is very good in Fig. 2, within the statistical error of simulations, which is smaller than $5 \%$. It should be also mentioned that for very large times $t \gg t_{h}=b^{N-1} / \nu_{0}$ diffusion and transport become normal. However, since $t_{h}$ grows exponentially with $N$ this truly asymptotic regime can be made numerically not reachable and thus completely irrelevant, as in our simulations. When we speak about asymptotic regime ' $t \rightarrow \infty$ ', we mean that time $t$ is large but yet much smaller than $t_{h}$, i.e. still within the regime of anomalous diffusion and transport. However, the obtained results correspond to the true $t \rightarrow \infty$ limit within the FLE description.
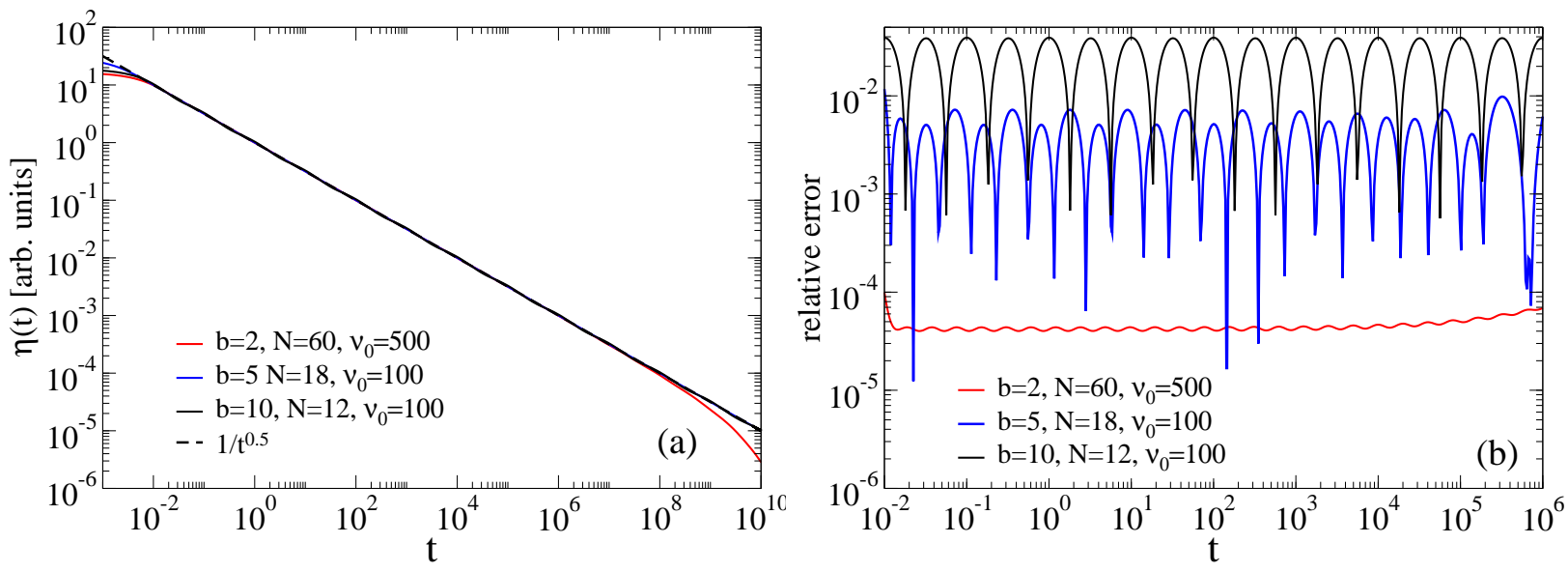

Figure 1. (a) Power law kernel, $1 / t^{\alpha}$, vs. its approximation by a sum of exponentials, $C_{\alpha}(b) \sum_{i=1}^{N} \nu_{i}^{\alpha} \exp \left(-\nu_{i} t\right), \nu_{i}=\nu_{0} / b^{i-1}$, for $\alpha=0.5$ and different $b, \nu_{0}$, and $N$, with $C_{0.5}(10)=1.3, C_{0.5}(5)=0.909, C_{0.5}(2)=0.39105$; (b) relative error of the corresponding approximations.

\subsection{Anomalous ratchet}

We use the outlined approach to study anomalous fluctuation-induced transport in periodic ratchet potentials $U(x)$ lacking space-inversion symmetry, i.e. $U(x+L)=U(x)$, where $L$ is spatial period, but 
$U(-x) \neq U\left(x+x_{0}\right)$, for any shift $x_{0}$, and driven by a periodic force with period $\mathcal{T}, f_{\text {ext }}(t+\mathcal{T})=f_{\text {ext }}(t)$. In this paper, $f_{\text {ext }}(t)=A \cos (\Omega t), \Omega=2 \pi / \mathcal{T}$.

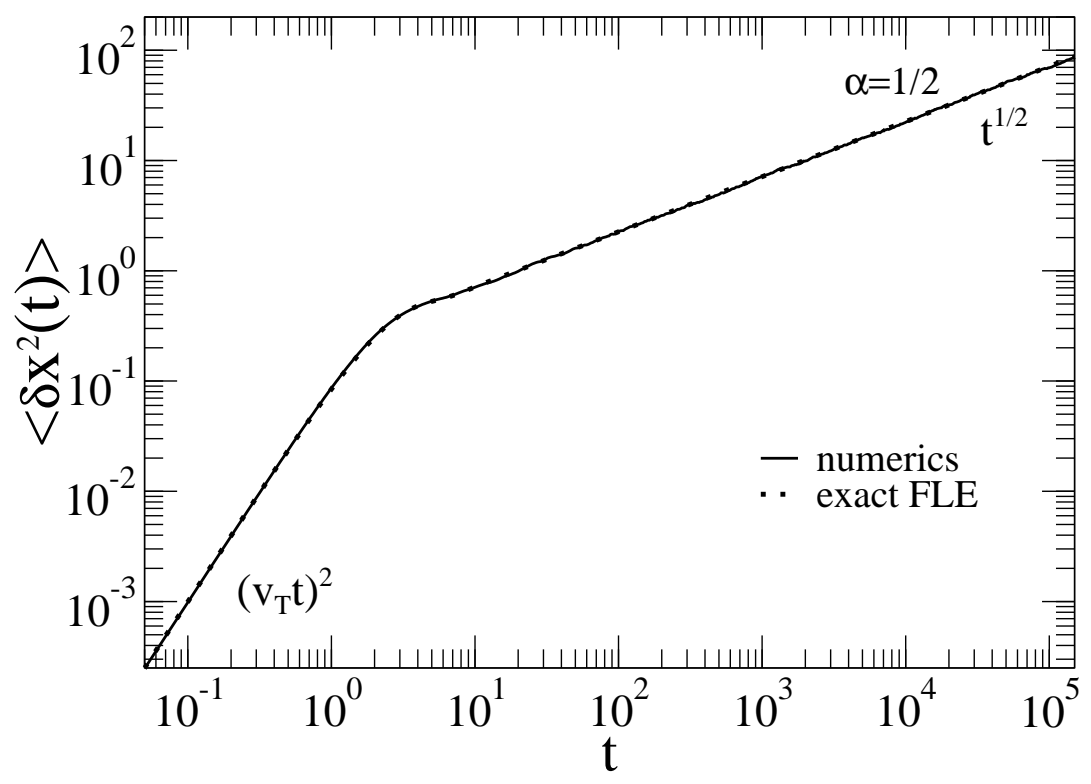

FiguRE 2. Numerics vs. exact result for the FLE dynamics without potential and for $T=0.1$. Other parameters are given in the text. The discrepancy is less than $5 \%$ even for large $t$ in this figure. Initially diffusion is always ballistic, as a manifestation of the inertial effects. It changes into subdiffusion on the time scale $t \gg 1$ exceeding much characteristic time of initial positive velocity autocorrelations defined by $\tau_{v}$. Slowly decaying negative velocity autocorrelations yield subdiffusion.

Moreover, a constant biasing force $f_{0}$ (load) can be applied in the direction opposite to the direction of rectified motion. The total potential is $V(x)=U(x)-x f_{\text {ext }}(t)+x f_{0}$. We are interested in the subtransport which can be characterized by the mean subvelocity

$$
v_{\alpha}=\Gamma(1+\alpha) \lim _{t \rightarrow \infty} \frac{\langle x(t)\rangle}{t^{\alpha}} .
$$

The subdiffusion coefficient is defined in a similar manner,

$$
D_{\alpha}=\Gamma(1+\alpha) \lim _{t \rightarrow \infty} \frac{\left\langle\delta x^{2}(t)\right\rangle}{2 t^{\alpha}}
$$

where $\left\langle\delta x^{2}(t)\right\rangle$ is the position variance, $\left\langle\delta x^{2}(t)\right\rangle=\left\langle x^{2}(t)\right\rangle-\langle x(t)\rangle^{2}$. It should be mentioned that the normal velocity (with $\alpha=1$ ) is zero, $v_{1}=0$. This does not mean, however, that the particles are localized and the transport is absent. In the absence of a periodic driving and/or bias, there cannot be any directed transport, including anomalous one. It is forbidden by the symmetry of thermal detailed balance [40,45, 50]. Transport cannot emerge also within a linear response regime of periodic driving. However, it can emerge already as a quadratic response to such a driving [50]. Indeed, this is the case also for anomalous transport. Generally, the emergence of such a rectification current presents a strongly nonlinear and nonequilibrium effect. If driving is subthreshold, then a particle initially localized in a potential well 
would remain trapped in this potential well in the absence of thermal noise, for a nonresonant driving and sufficiently strong friction. Thermal fluctuations help to overcome the potential barrier and induce directional transport of particles. In the case of normal diffusion such a thermal fluctuation-assisted transport is optimized in the regime of adiabatically slow driving [4]. Very different from that, the anomalous subtransport is strongly suppressed for slow driving [22]. The physical mechanism behind this nontrivial result is rooted in the asymptotic insensitivity of both the viscoelastic subdiffusion and subtransport to the presence of periodic potential as it was discovered in Refs. [21,23,24]. The physical reason for this astonishing feature can easily be understood by splitting the thermal fractional Gaussian noise into two parts, fast and slow, doing this self-consistently with respect to a characteristic time to escape out of the potential well. The latter time always exists for the considered type of the anomalous stochastic dynamics. Fast noise components lead to the formation of a non-Markovian rate (they kick out the particle out of potential well), while the slow ones slowly modulate this rate in time. Namely, this slow modulation leads to the emergence of a large scale anti-persistency in the motion and determines anomalous character of the asymptotic dynamics. For very high potential barriers, the escape kinetics tends to the normal exponential one, even though it remains highly dispersive for the barriers smaller than about $10 k_{B} T$, depending on $\alpha$ [21]. Surprisingly, the escape events are not transport limiting for such anomalously slow viscoelastic transport. Subdiffusion cannot become faster in the presence of unbiased potential than in its absence, and the asymptotic limit of potential-free subdiffusion is attained. This feature of viscoelastic subdiffusion is very different from the case of CTRW subdiffusion and fractional Fokker-Planck dynamics [47] in washboard potentials that has been studied in $[18,28]$. The given heuristic explanation is not only validated by stochastic numerical simulations in Refs. [21,23,24], but is also supported by two different analytical theories of quantum dissipative dynamics coupled to sub-Ohmic thermal bath $[8,59]$. The latter one presents a quantum generalization and counterpart of the classical FLE dynamics. These different quantum theories were worked out only for a cosinusoidal potential $U(x)$ and only within a linear response regime of transport. The results in Refs. [21,23,24] imply, however, that this remarkable feature (i) is purely classical, i.e. no quantum-mechanical effects such as tunneling are involved, (ii) valid beyond the linear response regime, (iii) is not restricted by cosinusoidal potentials but is valid for arbitrary periodic potentials including ratchet potentials. In other terms, it is a truly universal feature, or universality class of viscoelastic subdiffusion.

This universal property is responsible for vanishing rectification ratchet effect in the limit $\mathcal{T} \rightarrow \infty$. However, for a constant driving force the transition to this asymptotic limit is generally very slow and depends strongly on the amplitude of the periodic potential. This makes the anomalous subdiffusive ratchets possible. Moreover, there exists an optimal driving frequency since the rectification effect vanishes also in the limit of a very fast driving. It has been shown recently [26] for the ratchet potential

$$
U(x)=-U_{0}[\sin (2 \pi x / L)+0.25 \sin (4 \pi x / L)]
$$

and harmonic driving, $f_{\text {ext }}(t)=A \cos (\Omega t)$, that this optimization can be related to a stochastic resonance (SR) effect [12], i.e. synchronization of the thermally activated jumps over one or several potential wells with the half-period of the potential tilt in the transport direction. Such a non-Markovian SR [15-17] is clearly impossible for anomalous dynamics based on divergent mean residence times. Moreover, the considered dynamics fully retains inertial effects which can lead to weakly damped coherent oscillations within one potential well, so that the conventional resonance is, in principle, also possible.

The role of the inertial effects can be characterized by the dimensionless parameter $r_{v}=1 /\left(\omega_{0} \tau_{v}\right)$, where $\omega_{0}=(2 \pi / L)\left(3^{3 / 8} / 2^{1 / 4}\right) \sqrt{U_{0} / m}$ is the bottom and (imaginary) top oscillation frequency in the considered potential at $A=0, f_{0}=0$, within the harmonic approximation and in the absence of friction. The inertial effects can only be negligible for $r_{v} \ll 1$ and not too small $\alpha$ [7]. The borderline value of $U_{0}$ corresponding to $r_{v}=1$ is in the dimensionless units $U_{0}^{*} \approx 0.0157$. For all $U_{0}$ larger than this value, like in this work, the inertial effects are very essential. The corresponding frequency of damped oscillations $\omega_{d}$ can be found as the imaginary part of the complex conjugated roots of the equation $s^{2}+\gamma_{\alpha} s^{\alpha}+\omega_{0}^{2}=0$, where $\gamma_{\alpha}=\eta_{\alpha} / \mathrm{m}$. From this in the used time scaling we obtain: $\omega_{d} \approx 4.17$ for $U_{0}=0.25, \omega_{d} \approx 5.79$ 
for $U_{0}=0.50$, and $\omega_{d} \approx 7.04$ for $U_{0}=0.75$. For the driving frequencies much smaller than $\omega_{d}$, as in this work, the conventional resonance effects are excluded, see but in Ref. [33] for the case of flashing anomalous ratchets, where they do play a profound role.

Previously, we have shown that anomalous ratchet transport optimizes at certain driving frequencies for a fixed driving amplitude, potential amplitude, and temperature [22]. It also optimizes versus temperature, for a fixed potential amplitude, driving amplitude, and frequency [26], as also Fig. 3 illustrates. Subvelocity shows a typical SR dependence $v_{\alpha}(T) \propto T^{-n} \exp (-\Delta U / T)$ [12] with maximum at $T_{\max }=\Delta U / n . \Delta U$ and $n$ are considered here as fitting parameters which deviate from the lowest second order response values $\Delta U=2 U_{0}$ and $n=2$.

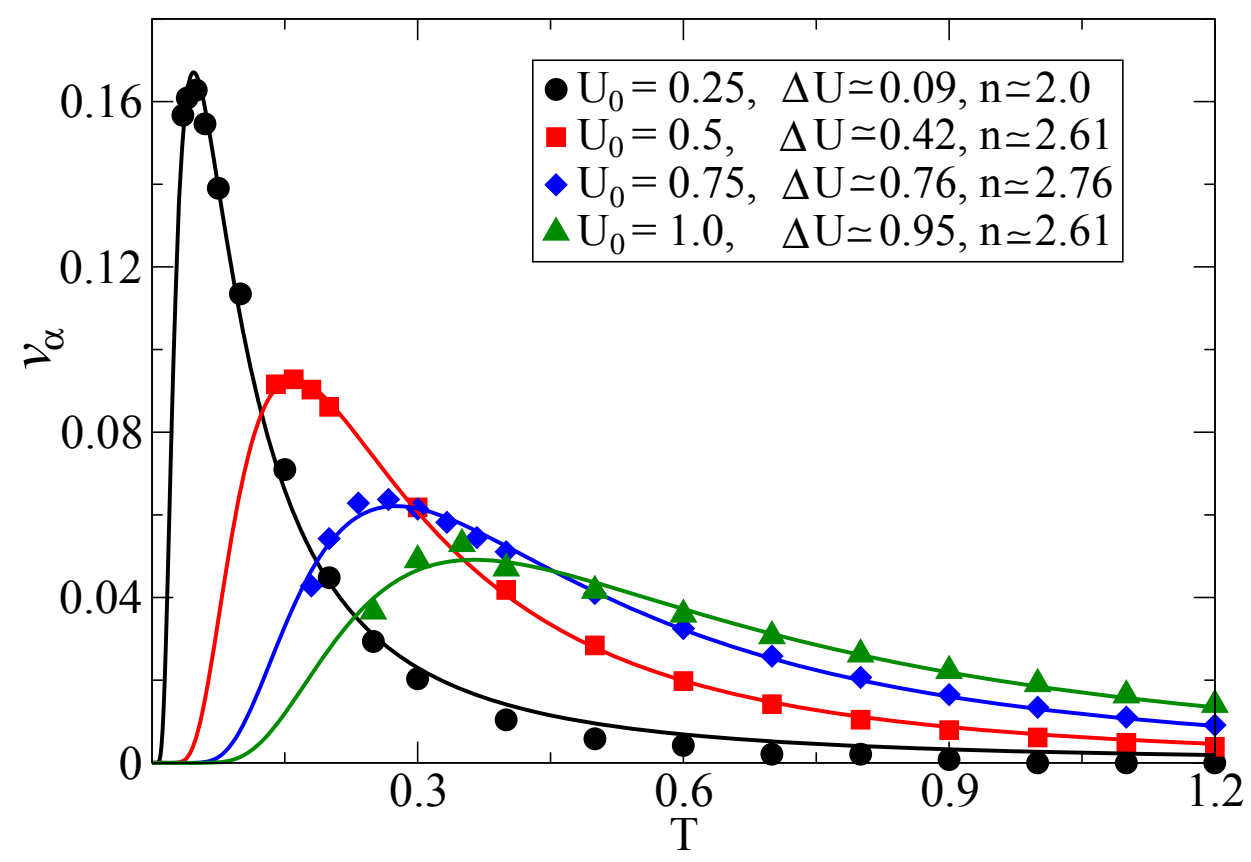

Figure 3. Subvelocity $v_{\alpha}$ vs. temperature for $A=0.8$ and $\Omega=0.1$ and different potential amplitudes $U_{0}$.

It should be also mentioned that subdiffusion remains asymptotically weakly sensitive to periodic driving. The subdiffusion coefficient $D_{\alpha}$ does not significantly deviate from its potential and driving free value $D_{\alpha}=T[22,26]$. This means that for low temperatures and small $U_{0}$ the transport can be low-dispersive and characterized by a large value of the generalized Peclet number $\mathrm{Pe}_{\alpha}:=v_{\alpha} L / D_{\alpha}$. The latter quantity measures the coherence quality of the anomalous transport. Such a remarkable degree of coherence is also very different from the alternative description of subdiffusion which is highly dispersive and featured by zero value of $\mathrm{Pe}_{\alpha}[24]$.

Furthermore, optimization occurs also with respect to driving strength. Indeed, for small $A, \nu_{\alpha} \propto A^{2}$. However, for $A \gg A_{1}=3 \pi U_{0} /(2 L)$, where $A_{1}$ is the critical value of a static forward tilt at which the potential barrier in the forward direction disappears, the transport should be suppressed. Therefore, one expects that it will be optimal for the values of $A$ around $A_{1}$. Indeed, numerics in Fig. 4 are consistent with this prediction.

The transport should also be optimized with the potential amplitude $U_{0}$ for other parameters kept fixed. The numerics in Fig. 5 reveal this clearly too. One can conclude that anomalous ratchet transport can indeed be optimized with respect to any of parameters $U_{0}, T, A, \Omega$. 


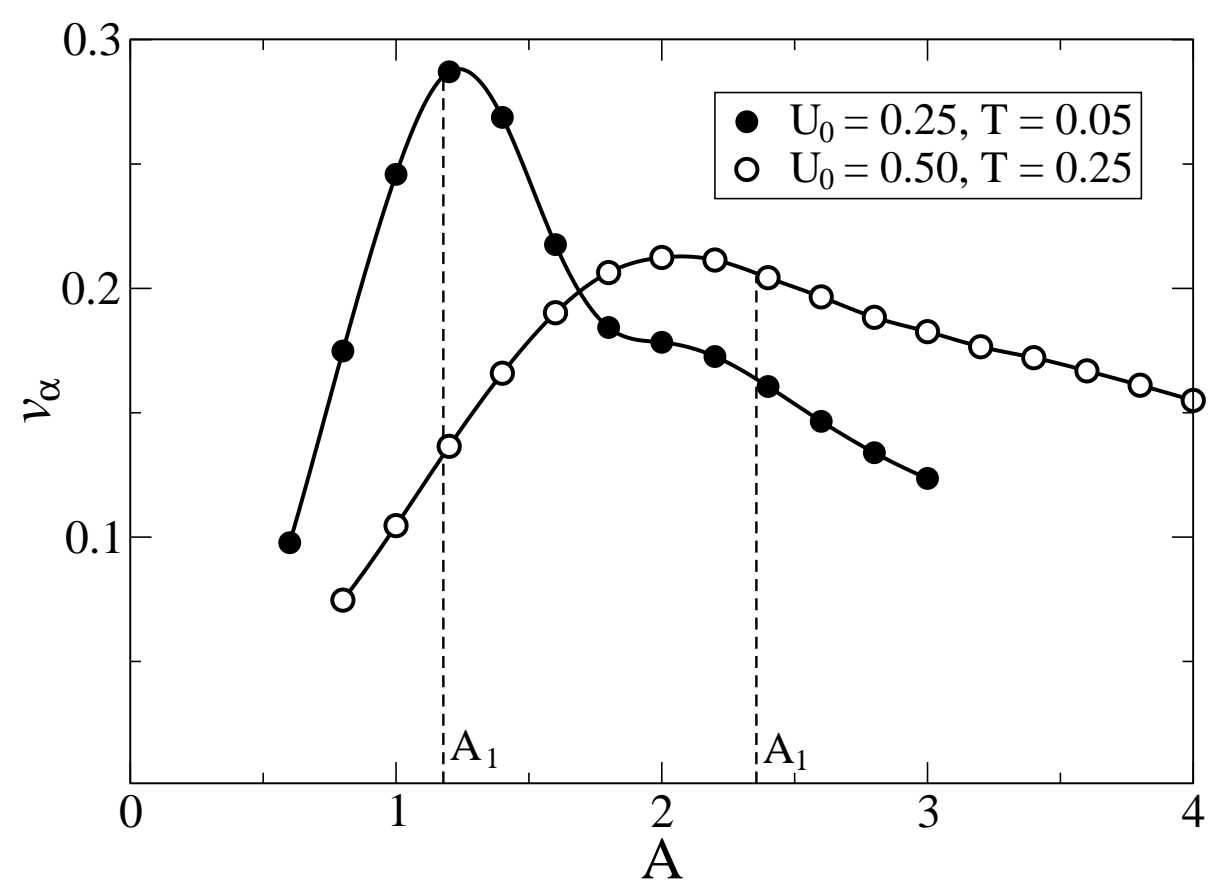

Figure 4. Subvelocity $v_{\alpha}$ as a function of the driving force amplitude $A$ for $\Omega=0.1$ and different values of the potential height $U_{0}$ and temperature $T$.

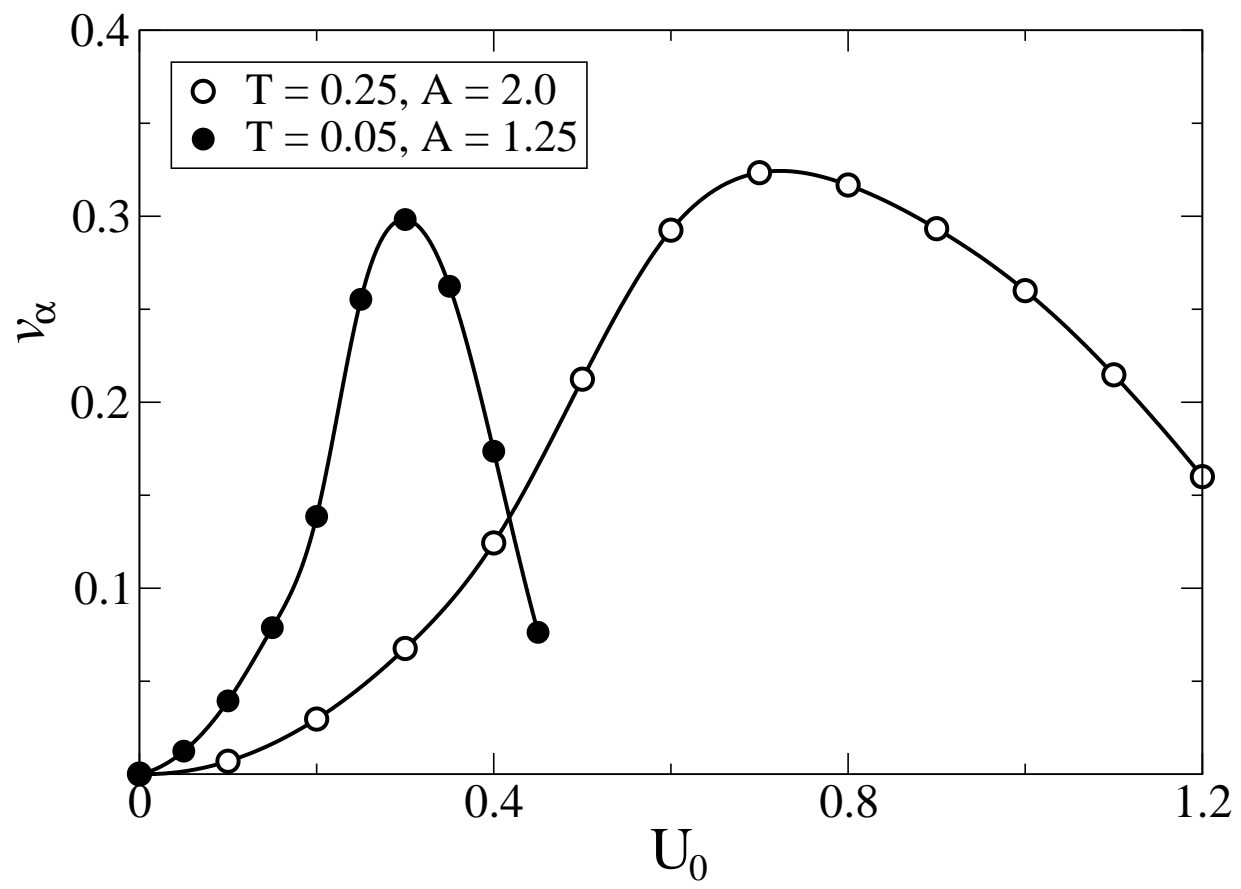

FiguRE 5. Subvelocity $v_{\alpha}$ as a function of the potential height $U_{0}$ at $\Omega=0.1$ and different values for driving amplitude $A$ and temperature $T$. 


\section{Thermodynamic efficiency of anomalous ratchets}

The great interest presents also the dependence of the transport subvelocity $v_{\alpha}$ on the load $f_{0}$ in the direction opposite to transport and the thermodynamic efficiency of such a transport. The first question has been answered recently in Ref. [26]. For a broad range of parameters the dependence of subvelocity on load turns out to be very simple,

$$
v_{\alpha}\left(f_{0}\right) \approx v_{\alpha}(0)-f_{0},
$$

in the scaled dimensionless units. It must be stressed that the existence of a stopping force $f_{\text {cr }}$ such that $v_{\alpha}\left(f_{\text {cr }}\right)=0$ makes it clear that we are dealing with a genuine ratchet effect to distinguish it from pseudo-ratchets, e.g. dissipationless quantum ratchets, which are featured by an infinitely small stopping force and therefore cannot do any useful work. We shall answer below the second question, which was not addressed thus far for the anomalous ratchet transport. Here we follow to the approach by Sekimoto [53], and others [32] developed for the normal diffusion ratchets. Our way of Markovian embedding makes its application to anomalous ratchets straightforward.

Indeed, let us multiply the second equation in $(2.2)$ by $v(t)$, integrate and average it over the trajectory realizations. The following energy balance equation follows:

$$
\Delta E_{\text {int }}(t)=E_{\text {pump }}(t)-W(t)+\Delta Q(t),
$$

where $\Delta E_{\text {int }}(t)=E_{\text {int }}(t)-E_{\text {int }}(0)$ is the change of internal energy of the Brownian motor particle, $E_{\text {int }}(t)=\left\langle m v^{2}(t) / 2+U(x(t))\right\rangle$, within the time interval $[0, t), E_{\text {pump }}(t)=\int_{0}^{t}\left\langle f_{\text {ext }}\left(t^{\prime}\right) v\left(t^{\prime}\right)\right\rangle d t^{\prime}$ is the energy pumped by the external force $f_{\text {ext }}(t), W(t)=f_{0}\langle x(t)-x(0)\rangle$ is the useful work done against the load $f_{0}$, and $\Delta Q(t)=\sum_{i=1}^{N} \int_{0}^{t}\left\langle u_{i}\left(t^{\prime}\right) v\left(t^{\prime}\right)\right\rangle d t^{\prime}$ is the heat exchanged with the environment. Obviously, $\Delta E_{\text {int }}(t)$ is bounded and it can be neglected in the energy balance for large $t$. The efficiency of such an isothermal motor can be defined as $R(t)=W(t) / E_{\text {pump }}(t)$. In principle (but not for the system considered!), it can arrive at the theoretical maximum of one if isothermal motor operates near to the thermal equilibrium, where the heat exchange with the thermal reservoir is absent, $\Delta Q \rightarrow 0$. Then, however, also the operating power vanishes, $P_{W}(t)=W / t \rightarrow 0$, i.e. the motor operates at zero power because of its operational speed is also close to zero. Recent theoretical work shows that the maximal efficiency of isothermal engines at maximal power can even exceed 50\% [58] as suggested in [52]. The efficiency of normal diffusion rocking ratchets is much lower, a few per cents only, at best. Subdiffusion introduces new features. Namely, since the useful work done against the load scales linearly with the traveling distance, it scales sublinearly with time, $W(t)=a_{W} t^{\alpha}$, where $a_{W}=f_{0} v_{\alpha}\left(f_{0}\right) / \Gamma(1+\alpha)$, within the regime of subdiffusion. Therefore, the working power $P_{W}$ decays algebraically in time, $P_{W} \propto 1 / t^{1-\alpha}$. Like the concept of normal average velocity is modified for subvelocity in the case of subtransport, one should modify also the concept of power accordingly. Let us do this by defining sub-power, $P_{\alpha, W}:=\Gamma(1+\alpha) W(t) / t^{\alpha}$, instead of the work produced per unit of time. In our notation, $P_{\alpha, W}=\Gamma(1+\alpha) a_{W}=f_{0} v_{\alpha}\left(f_{0}\right)$. However, the energy pumped into the system by the driving force scales linearly with time, $\Delta E_{\text {pump }}(t)=a_{E} t$, as numerical results show. This is also an expected result. Moreover, the pumping power $a_{E}$ does not depend on load, see in Fig. 6. This is similar to the normal diffusion case [53]. Therefore, the efficiency $R(t)$ declines algebraically in time as $R(t)=a_{R} / t^{1-\alpha}$, and for the coefficient $a_{R}$ we obtain, upon taking into account Eq. (3.1), a very simple result:

$$
a_{R} \approx f_{0}\left[v_{\alpha}(0)-f_{0}\right] /\left[a_{E} \Gamma(1+\alpha)\right] .
$$

The maximal $a_{R}$ is achieved at $f_{\mathrm{opt}}=v_{\alpha}(0) / 2$, i.e. at the half of the stopping force $f_{\max }=v_{\alpha}(0)$. Clearly, the efficiency is zero both for zero load and the maximal load, and the maximal $a_{R}$ is $a_{R}^{(\max )}=$ $v_{\alpha}^{2}(0) /\left[4 a_{E} \Gamma(1+\alpha)\right]$. This simple theory agrees with the numerical results in Fig. 6 very well. The efficiency decays algebraically slow and on appreciably long time scale it can compare well with the efficiency of normal diffusion ratchets. Indeed, the maximal efficiency in Fig. 6 for $t=1$ is about $30 \%$, and for $t=100$ it is still $3 \%$, reducing to $0.03 \%$ at the end point $t_{\max }=10^{6}$ in our simulations. In this 
respect, the first paper on the efficiency of normal diffusion rocking ratchets reported on the efficiencies as small as $0.01 \%$ [53]. If $\alpha$ is closer to one, e.g. $\alpha=3 / 4$, as for diffusion in solutions of semi-flexible polymers [1], then the efficiency decays even slower, as $1 / t^{1 / 4}$.

The real molecular motors do not operate as rocking ratchets, but rather as flashing ones with the minimum of the potential which is displaced in the transport direction at each flash [32,42]. The efficiency of such molecular motors can be very high, close to maximally possible [32,42]. How operating in such viscoelastic media as cytosol of biological cells influences the efficiency of biological molecular motors is an intriguing issue which we currently investigate. The results of this work point out into direction that the efficiency of molecular motors can be also high in the viscoelastic environments, on the relevant mesoscopic space/time scales.

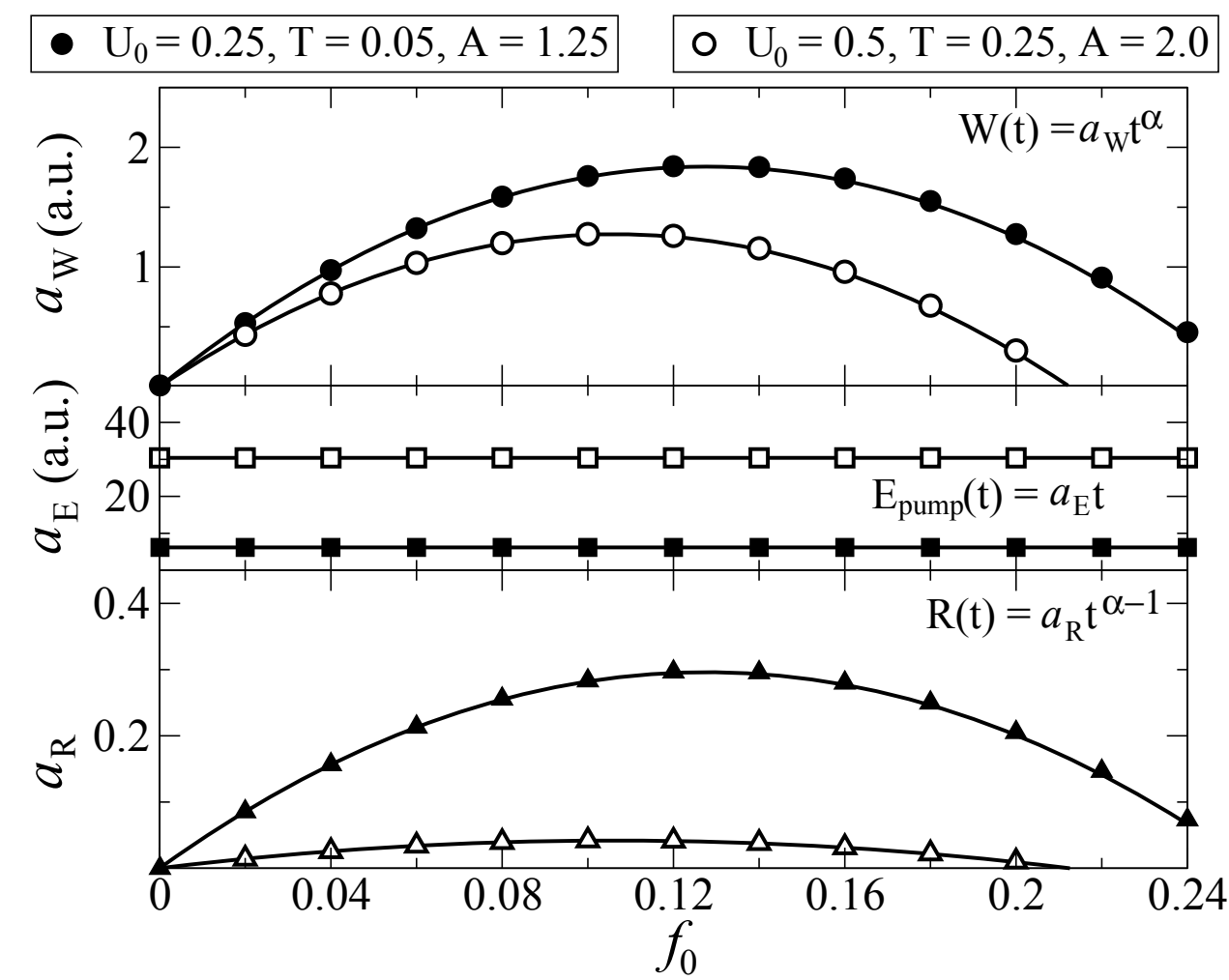

Figure 6 . Dependencies of the coefficients for the useful work $a_{W}$, input energy $a_{E}$ and the efficiency $a_{R}$ versus load for two different sets of parameters and a fixed value $\Omega=0.1$. The symbols depict the numerical results and the solid lines show the corresponding analytical predictions.

\section{Summary and Conclusions}

In this work, we investigated anomalous ratchet effect within the Generalized Langevin Equation approach to subdiffusion. Physically the origin of such a subdiffusion is rooted in the phenomenon of viscoelasticity which leads to the emergence of long-range persistent anticorrelations in the Brownian particle displacements and also to negative correlations of its velocity fluctuations. The power-law-decaying viscoelastic memory kernel corresponds to the sub-Ohmic model of the thermal bath [59] within the dynamical ap- 
proach to generalized Brownian motion, where the thermal environment is modeled by a quasi-infinite set of harmonic oscillators. In the inertialess limit, or on the time scale exceeding much the time scale of relaxation of the velocity variable and in the absence of external forces or for a constant force, this model yields fractional Brownian motion and therefore explains its dynamical origin. Moreover, it is known [20] that the dynamical response of such a motion trapped in parabolic potentials is given, in neglecting the inertial effects, by the famous Cole-Cole expression [9]. Such a response is indeed commonly measured in viscoelastic complex liquids and in glass-like media. It corresponds to the $\beta$-relaxation. This provides an additional physical justification for the model considered. The corresponding memory kernel can also be efficiently approximated by a sum of exponentials reflecting a hierarchy of viscoelastic modes of environment. We used this fact for a highly efficient Markovian embedding of the considered non-Markovian GLE dynamics with long-range memory. This methodology allows also for a vivid physical interpretation. Namely, the medium is represented by a set of inertialess Brownian quasi-particles elastically coupled to the moving Brownian particle (see video on the web site of journal). Fast medium's deformation (represented by rapidly relaxing quasi-particles) follows immediately to the Brownian particle, which effectively becomes dressed with these fast Brownian particles. This reminds the small polaron concept in condensed matter physics. However, the slow deformations cannot immediately follow to the particle. Their retarded motion provides a physical mechanism for slowly decaying memory about the former positions of the Brownian particle and causes subdiffusion on a long time scale. The presence of a periodic potential influences the motion of Brownian particle, but it does not affect directly the medium. As a result, the dynamics of slow deformations is not affected by the presence of potential and this leads to the universality class of viscoelastic subdiffusion and transport in tilted periodic potentials. Here, the asymptotic behavior of the Brownian particles does not depend on the presence of periodic potential. For this reason, the anomalous ratchet effect is not possible for adiabatically slow rocking. However, it emerges for a sufficiently fast driving. Moreover, it optimizes when the driving period matches a characteristic time scale of the temperature activated transitions to the neighboring potential wells. This leads to the optimization of anomalous ratchet transport with respect to different parameters: frequency and amplitude of the time-periodic driving force, potential amplitude, and temperature. The last three optimizations were addressed in this paper. We studied also the problem of thermodynamic efficiency of such anomalous isothermal Brownian motors and proposed a simple theory which remarkably well agrees with the numerical results. Most importantly, since the position of Brownian particle scales sublinearly with time, one cannot characterize such anomalous Brownian motors using the conventional notion of working power, since the useful work done by motors against a load scales also sublinearly with time. Instead, we introduced the notion of sub-power and showed that the efficiency of such motors decays algebraically slow in time since the energy pumped into the directed motion scales with time linearly. Together with our other recently published works this contribution opens the new field of anomalous Brownian motors which can especially be relevant for transport processes in living cells, in a more realistic setup of flashing ratchets pertinent to biological molecular motors [32]. This future direction grows naturally from the research already done. It will be explored in the nearest future.

Acknowledgements. Support of this research by the Deutsche Forschungsgemeinschaft, Grants GO 2052/1-1 and GO 2052/1-2 is gratefully acknowledged.

\section{References}

[1] F. Amblard, A. C. Maggs, B. Yurke, A. N. Pargellis, S. Leibler. Subdiffusion and anomalous local viscoelasticity in actin networks. Phys. Rev. Lett. 77 (1996), 4470-4473.

[2] F. Barbi, M. Bologna, P. Grigolini. Linear response to perturbation of non-exponential renewal processes. Phys. Rev. Lett., 95 (2005), 220601.

[3] E. Barkai. Fractional Fokker-Planck equation, solution, and application. Phys. Rev. E, 63 (2001), 046118.

[4] R. Bartussek, P. Hänggi, J. G. Kissner. Periodically rocked thermal ratchets. Europhys. Lett., 28 (1994), 459-464. 
[5] N. N. Bogolyubov, Elementary example of establishing thermal equilibrium in a system coupled to thermostat. in On some statistical methods in mathematical physics. Acad. Sci. Ukrainian SSR, Kiev, 1945, pp. 115-137, in Russian.

[6] J.-P. Bouchaud, A. Georges. Anomalous diffusion in disordered media: statistical mechanisms, models and physical applications. Phys. Rep., 195 (1990), 127-293.

[7] S. Burov and E. Barkai, Critical exponent of the fractional Langevin equation. Phys. Rev. Lett., 100 (2008), 070601.

[8] Y. C. Chen, J. L. Lebowitz. Quantum particle in a washboard potential. I. Linear mobility and the Einstein relation. Phys. Rev. B, 46 (1992), 10743-10750.

[9] K. S. Cole, R. H. Cole. Dispersion and absorption in dielectrics I. Alternating current characteristics. J. Chem. Phys., 9 (1941), 341-352.

[10] W. H. Deng, E. Barkai, Ergodic properties of fractional Brownian-Langevin motion. Phys. Rev. E, 79 (2009), 011112.

[11] G. W. Ford, M. Kac, P. Mazur. Statistical Mechanics of Assemblies of Coupled Oscillators. J. Math. Phys., 6 (1965), No. 4, 504-515.

[12] L. Gammaitoni, P. Hänggi, P. Jung, F. Marchesoni. Stochastic Resonance. Rev. Mod. Phys., 70 (1998), $223-288$.

[13] T. C. Gard. Introduction to Stochastic Differential Equations. Dekker, New York, 1988.

[14] A. Gemant. A method of analyzing experimental results obtained from elasto-viscous bodies., Physics, 7 (1936), $311-317$.

[15] I. Goychuk, P. Hänggi, Non-Markovian stochastic resonance. Phys. Rev. Lett., 91 (2003), 070601.

[16] I. Goychuk, P. Hänggi, Theory of non-Markovian stochastic resonance. Phys. Rev. E, 69 (2004), 021104.

[17] I. Goychuk, P. Hänggi, J. L. Vega, S. Miret-Artes. Non-Markovian stochastic resonance: three state model of ion channel gating. Phys. Rev. E, 71 (2005), 061906.

[18] I. Goychuk, E. Heinsalu, M. Patriarca, G. Schmid, P. Hänggi. Current and universal scaling in anomalous transport. Phys. Rev. E, 73 (2006), 020101 (Rapid Communication).

[19] I. Goychuk and P. Hänggi. Anomalous escape governed by thermal 1/f noise., Phys. Rev. Lett., 99(2007), 200601.

[20] I. Goychuk, Anomalous relaxation and dielectric response. Phys. Rev. E, 76 (2007), 040102(R).

[21] I. Goychuk. Viscoelastic subdiffusion: from anomalous to normal. Phys. Rev. E, 80 (2009), 046125.

[22] I. Goychuk. Subdiffusive Brownian ratchets rocked by a periodic force. Chem. Phys., 375 (2010), $450-457$.

[23] I. Goychuk, P. Hänggi. Subdiffusive dynamics in washboard potentials: two different approaches and different universality classes. in J. Klafter, S. C. Lim, R. Metzler, editors. Fractional Dynamics, Recent Advances. World Scientific, Singapore, 2011, Ch. 13, pp. 307-329.

[24] I. Goychuk. Viscoelastic subdiffusion: generalized Langevin equation approach. Adv. Chem. Phys., 150 (2012), $187-253$.

[25] I. Goychuk. Is subdiffusional transport slower than normal? Fluct. Noise Lett., 11 (2012), 1240009

[26] I. Goychuk, V. Kharchenko. Fractional Brownian motors and stochastic resonance. Phys. Rev. E, 85 (2012), 051131.

[27] Y. He, S. Burov, R. Metzler, E. Barkai. Random time-scale invariant diffusion and transport coefficients. Phys. Rev. Lett., 101 (2008), 058101.

[28] E. Heinsalu, M. Patriarca, I. Goychuk, G. Schmid, P. Hänggi. Fractional Fokker-Planck dynamics: Numerical algorithm and simulations. Phys. Rev. E, 73 (2006), 046133.

[29] E. Heinsalu, M. Patriarca, I. Goychuk, P. Hänggi, Use and abuse of a fractional Fokker-Planck dynamics for timedependent driving. Phys. Rev. Lett., 99 (2007), 120602.

[30] E. Heinsalu, M. Patriarca, I. Goychuk, P. Hänggi. Fractional Fokker-Planck subdiffusion in alternating force fields. Phys. Rev. E, 79 (2009), 041137.

[31] B. D. Hughes. Random walks and random environments, Vols. 1,2. Clarendon Press, Oxford, 1995.

[32] F. Jülicher, A. Ajdari, J. Prost. Modeling molecular motors. Rev. Mod. Phys., 69 (1997), 1269-1282.

[33] V. Kharchenko, I. Goychuk. Flashing subdiffusive ratchets in viscoelastic media. New J. Phys., 14 (2012), 043042.

[34] A. N. Kolmogorov. Dokl. Akad. Nauk SSSR, 26 (1940), 115-118 (in Russian), English transl. Wiener spirals and some other interesting curves in a Hilbert space, in V. M. Tikhomirov, editor. Selected Works of A. N. Kolmogorov, vol. I, Mechanics and Mathematics. Kluwer, Dordrecht, 1991, pp. 303-307.

[35] R. Kubo. The fluctuation-dissipation theorem. Rep. Prog. Phys., 29 (1966), 255-284.

[36] R. Kubo, M. Toda, and M. Hashitsume. Nonequilibrium Statistical Mechanics, 2nd ed. Springer, Berlin, 1991.

[37] R. Kupferman. Fractional kinetics in Kac-Zwanzig heat bath models. J. Stat. Phys., 114 (2004), 291-326.

[38] A. Lubelski, I. M. Sokolov, J. Klafter. Nonergodicity mimics inhomogeneity in single particle tracking. Phys. Rev. Lett., 100 (2008), 250602.

[39] E. Lutz. Fractional Langevin equation. Phys. Rev. E, 64 (2001), 051106.

[40] M. O. Magnasco. Forced thermal ratchets. Phys. Rev. Lett., 71 (1993), 1477-1481.

[41] F. Mainardi, P. Pironi. The fractional Langevin Equation: Brownian motion revisited. Extracta Mathematicae, 11 (1996), 140

[42] Yu. A. Makhnovskii, V. M. Rozenbaum, D.-Y. Yang, S. H. Lin, T. Y. Tsong. Flashing ratchet model with high efficiency. Phys. Rev. E, 69 (2004), 021102.

[43] B. B. Mandelbrot, J. W. van Ness. Fractional Brownian motions, fractional noises and applications. SIAM Review, 10 (1968), No. 4, 422-437.

[44] B. B. Mandelbrot. The Fractal Geometry of Nature. W.H. Freeman Company, New York, 1977.

[45] P. Hänggi, F. Marchesoni. Artificial Brownian motors: bontrolling transport on the nanoscale. Rev. Mod. Phys., 81 (2009), 387-442.

[46] J. C. Maxwell. On the dynamical theory of gases. Phil. Trans. R. Soc. Lond., 157 (1867), 49-88. 
[47] R. Metzler, J. Klafter. The random walks guide to anomalous diffusion: a fractional dynamics approach. Phys. Rep., 339 (2000) 1-77.

[48] E. M. Montroll, G.H. Weiss. Random walks on lattices.2. J. Math. Phys., 6 (1965), No. 2, 167.

[49] R. G. Palmer, D. L. Stein, E. Abrahams, P. W. Anderson. Models of hierarchically constrained dynamics for glassy relaxation. Phys. Rev. Lett., 53 (1984), 958-961.

[50] P. Reimann. Brownian motors: noisy transport far from equilibrium. Phys. Rep., 361 (2002), 57-265.

[51] H. Scher, E. M. Montroll. Anomalous transit time dispersion in amorphous solids. Phys. Rev. B, 12 (1975), $2455-2477$.

[52] U. Seifert. Efficiency of autonomous soft nanomachines at maximum power. Phys. Rev. Lett., 106 (2011), 020601.

[53] K. Sekimoto. Kinetic characterization of heat bath and the energetics of thermal ratchet models. J. Phys. Soc. Jpn., 66 (1997) 1234-1237.

[54] M. F. Shlesinger. Asymptotic solutions of continuous time random walks. J. Stat. Phys., 10 (1974), $421-434$.

[55] I.M. Sokolov, J. Klafter. From diffusion to anomalous diffusion: a century after Einstein's Brownian motion. Chaos, 15 (2005), 026103.

[56] I. M. Sokolov, J. Klafter. Field-induced dispersion in subdiffusion. Phys. Rev. Lett., 97 (2006), 140602.

[57] I. M. Sokolov, E. Heinsalu, P. Hänggi, I. Goychuk. Universal fluctuations in subdiffusive transport. Europhys. Lett., 86 (2009), 30009.

[58] C. Van den Broeck, N. Kumar, K. Lindenberg. Efficiency of isothermal molecular machines at maximum power. Phys. Rev. Lett., 108, (2012) 210602.

[59] U. Weiss. Quantum Dissipative Systems, 2nd ed. World Scientific, Singapore, 1999.

[60] R. Zwanzig. Nonlinear generalized Langevin equations. J. Stat. Phys., 9 (1973), 215-220.

[61] R. Zwanzig. Nonequilibrium statistical mechanics. Oxford Univ. Press, Oxford, 2008. 